\title{
RFID Integrated Teacher Monitoring
}

\author{
Article by Adewopo Adeniyi \\ M.Sc, Texila American University, Nigeria \\ Email: preciousadewopon@yahoo.com
}

\section{Introduction}

Radio Frequency Identification (RFID) is a generic term for non-contacting technologies that use radio waves to automatically identify people or objects. There are several methods of identifying human or object, but the most common, in today's world, is to store a unique serial number that identifies a person or object on a microchip that is attached to an antenna. The combined antenna and microchip are called an "RFID transponder" or "RFID tag" and work in combination with an "RFID reader" (sometimes called an "RFID interrogator" because it queries the tag in the cause of retrieving the stored number or information). An RFID system consists of a reader and one or more tags. The reader's antenna is used to transmit radio frequency (RF) energy. Depending on the tag type, the energy is "harvested" by the tag's antenna and used to power up the internal circuitry of the tag. The tag will then modulate the electromagnetic waves generated by the reader in order to transmit its data back to the reader. The reader receives the modulated waves and converts them into digital data.

The RFID transponder are classified as either active, if it requires power, or pass if no power is required. The range and capability is improved when it is active but it tends to make the transponder expensive. Sometimes it cost is ten times that of a passive transponder. The passive transponders get it needed power from the radio energy emitted by the reader during the course of reading/querying. The transponders and readers come in various frequency ranges:

Low frequency: $125-134 \mathrm{KHz}$

High frequency: $13.36 \mathrm{MHz}$

Ultra high frequency: $902-928 \mathrm{MHz}$

Micro wave frequency: $2.4 \mathrm{GHz}$

\section{Related work}

Bolivar Torves, Qing Pang, Gordon W. Skelton, Scott Bridge (2013) described the research that integrated RFID and WSN technologies to design a real time access control application. Their purpose was to integrate RFID reader to a wireless sensor network to identify individuals' carrying RFID tags. The work of sending commands to the RFID to make it read a tag and send it back to the computer was accomplished by python code developed which also controls the data signal. One of the hardware employed in their work is SNAP SN171 Proto board. The RFID node of the board sends and receives signals from the RFID node each time it is told to do so by the application software. The node that starts the procedure of continuously detecting a tag near the RFID node is the polling node. This node sends a Remote Procedure call (RPC).

Md. Kafil Uddin, Mahbubur Rahman, Go Kim and BongHee Hong (2010) designed and implemented an efficient RFID sensor middleware architecture for sensor tag that incorporates extended APIs to provide compatibility and global interoperability of the system. Their purpose is to design and implement RFID sensor middleware compliant with EPC Architecture framework. The architecture had the application layer as the top layer and provided four APIs. The $4^{\text {th }}$ API is the sending alarm notification which is an API that sends synchronous reporting of sensor tag data for user query and alarm notification to the users synchronously of any alarm event.

Noureddine Chikouche, Foudil Cherif \&Mohamed Bennohammed (2012) proposed an authentication protocol based on the combination between RFID system and Biometric system. The purpose of the work is to verify secrecy, authentication of the tag and 
South American Journal of Academic Research

Special Edition May 2016

authentication of the reader by AVISPA and SPAN tools. In their work, they submitted that communication amongst the server and the reader and communication between the server and the sensor is secure. On the contrary, communication between the tag and the reader is not secured and it is based on radio frequency wave which adversaries can obscure, block, modify and inject messages from any communication between a reader and a tag.

Steffan Elmstrom Holst Jensen \& Rune Hylsberg Jacobsen (Retrieved 2015) wrote on more advanced active tags. Sensor tags may run additional software and can be equipped with communication software such as IP protocol stack. Another interesting use case can be applied to sensor tags. When these sensor tags connect to network sensor data can be retrieved from the tag.

Rashid Ahmed \& John N Avarissiotis (Retrieved 2015) collected data from special badges containing RFID tags and identified these tags to interact with NTUA information system to detect the employees that are entering and leaving the college.. The purpose is to identify employees using RFID in IE-NTUA. RFIDs can read information on multiple tags simultaneously without necessarily requiring losses and without the need for a particular orientation.

\section{Methodology}

In the proposed work, passive transponders will be used. When a passive transponder gets into the range of a reader, utilizing the released energy of the reader, it sends it identification to the reader. This process is in accordance with the Faraday's law of electromagnetic induction. The current flowing through the interrogator (reader) coil produces a magnetic field that links the coil of the transponder and causes current to flow in it. As the signal continues to flow, there is variation in the load on the antenna as a result of the modulation which is in accordance with the transmitted data/information.

The system will comprise of passive UHF tag (transponder card), a reader, microcontroller control unit and a wireless transceiver.

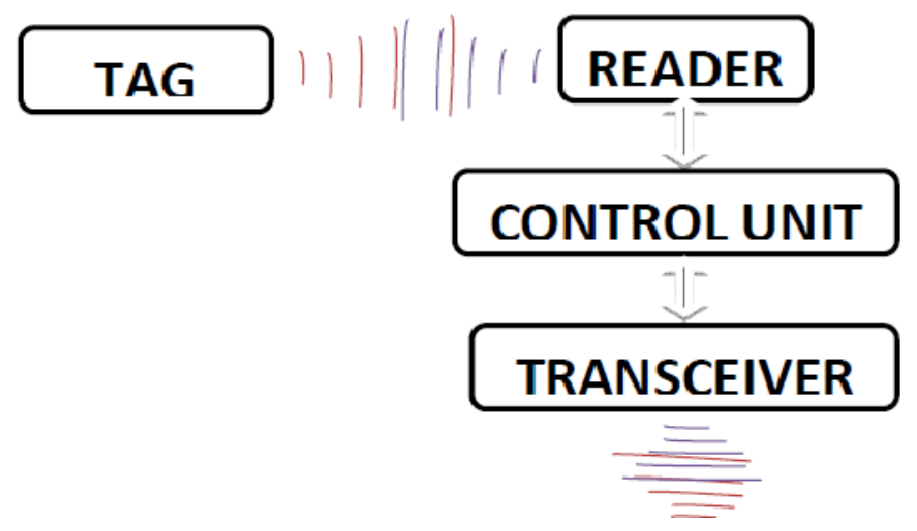

\section{Tag}

The tag for the monitoring system is a small card with a passive RFID chip implanted in them. This card carries the unique identification number of the holding teacher and shall be carried to the lass at all time by the teacher otherwise; the teachers' activities of the day will not be captured. Since the card requires no power for operation and no maintenance by the holder, there is no technical input required from the holding teacher. All the teacher needs to do is to carry the card in his pocket or hand bag. The card when it comes into any class where there is a reader, it communicates with the reader in that class wirelessly without the knowing of the holding teacher and very fast. The communication start and finish in less than one second of entering the vicinity of the reader. The card is made of plastic like the regular ATM card. 


\section{Reader}



RFID tag

The reader retrieves the information stored in the tag via electromagnetic energy emission. When the tag senses this emission, it sends it identification to the reader. The reader under the control of the control unit, forward the received information to the embedded microcontroller. In a passive RFID system, it is the reader that initiates the reading process. When the reader is down, the section covered by that reader is cut off from the system. The reader coverage is expected to cover the entire teacher space of the classroom. This is to ensure continues monitoring of the teacher through intermittent querying of the teacher's presence. This is done to capture the time the teacher leaves the class.

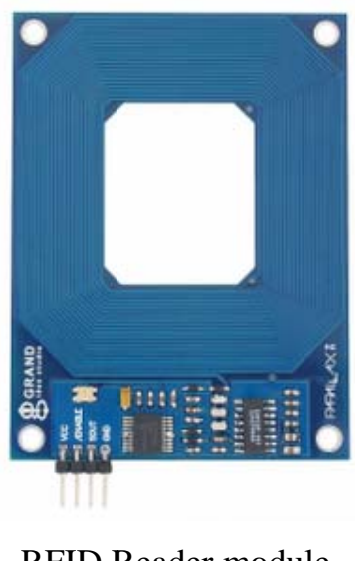

\section{Control unit}

The control unit controls the reading operations and data transmission and reception process. The heart of the control unit is PIC18F24200, a very powerful microcontroller with $16 \mathrm{~K}$ word program memory, 768 SRAM (static random access memory), 256 EEPROM (electrically erasable programmable read only memory), $25 \mathrm{i} / \mathrm{o}$ pins, SPI, $\mathrm{I}^{2} \mathrm{C}$, and EUSART communication modules suitable for communicating with a wireless transceiver like the UM92. The microcontroller is fast and can be clocked internally at $8 \mathrm{MHz}$ or externally up to $40 \mathrm{MHz}$. the control information is to written in C language and compiled and loaded into the microcontroller with a PIC microcontroller programmer. 


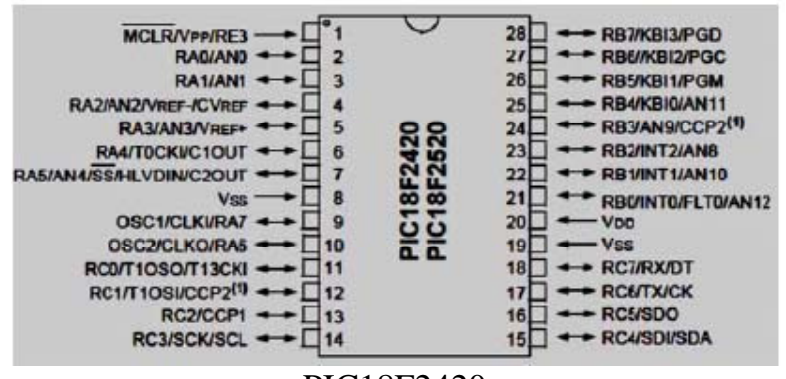

PIC18F2420

The microcontroller prompts the reader to send the id of the card in it vicinity every minute via the EUART (enhanced universal asynchronous receive transmit) port. When this information is received, the microcontroller sends it to the database station wirelessly through the wireless transceiver connected to it EUART port.

\section{Transceiver}

The transceiver is a wireless communication module that can receive and transmit at the same time. A typical module of this nature is the UM96.These small wireless modules use a seamless serial interface (UART) - whatever enters the TX pin at 9600bps shows up on the other unit's RX (database transceiver's) pin. All buffering and error detection is handled internally. And the best part - with $10 \mathrm{~mW}$ output, it reaches over 500 meters (half a kilometer range radius) sufficient to cover a normal school.

This unit is connected to the control unit's microcontroller TX and RX pins for incredibly simple serial cable replacement!

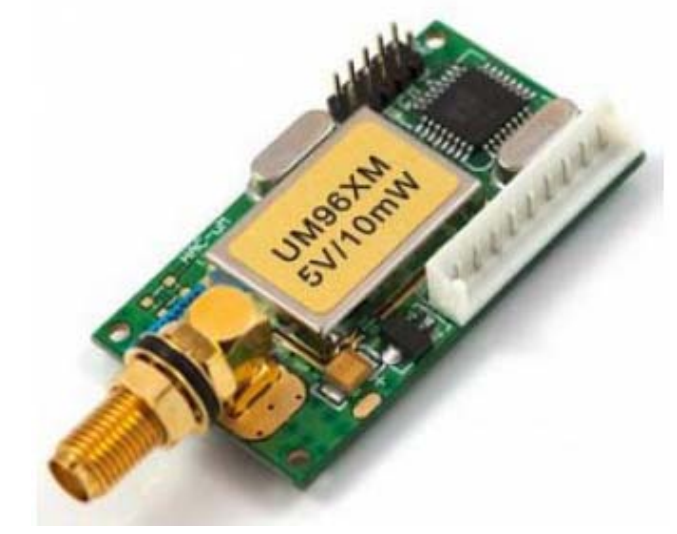

\section{Specifications:}

- Operating Voltage: 3.3V-5.5V

- 9600bps over 500m

- Modulation type: GFSK

- 8 Channels jumper selectable

- Low power : 30mA RX, 40mA TX, 20uA Sleep

- Small footprint size: 26 x $47 \mathrm{~mm}$

The receiving transceiver is identified with the accompanying address of the receiver. Data can be transmitted in byte or as set of bytes. Each of the um96 to be will be identified with a classroom number programmed into the control microcontroller of the class.

\section{Database transceiver}

The database transceiver is the transceiver on the database side of the system for wireless communication. It is also UM96 transceiver. It wireless links the reader side TX with the database side RX and vice versa. The data base side is the data entry and processing side of 
the system. This module communicates the received data to the computer via RS232 communication port and protocol. It has its own unique address on the system network.

\section{Database computer}

A program in visual basic, C\# or JAVA, will be written to collect this information and tabulate them in a defined order. With this, the teachers can be identified; the time he/she enters and leaves a class can be documented for a period. This documentation will be marched with the class time table. With this, when another teacher gets into a class that he or she has no business with at any time, the information sent to the data base as at that time will be ignored.

\section{Networking}

To cover the entire class room of the school with this technology, the RFID reader, controller and transceiver unit (classroom unit) shall be duplicated. Each of this unit shall have their unique identification number for differentiating them. The database unit is just one and all classroom units send their information to it.

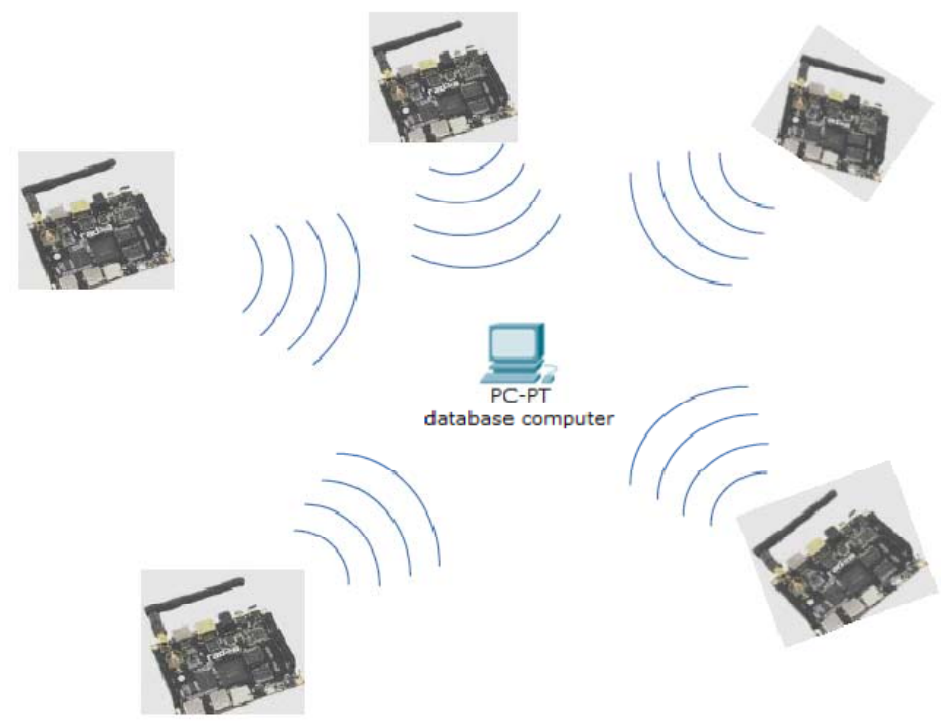

\section{Class room unit and database network}

\section{References}

[1.] Bolivar Torves, Qing Pang, Gordon W. Skelton, Scott Bridge (2013): “Integration of an RFID reader to a wireless sensor network and its use to identify an individual carrying tags“ In International Journal of Ad hoc sensor \& Ubiquitous computing Vol. 1, No.4

[2.] Md. Kafil Uddin, Mahbubur Rahman, Go Kim and BongHee Hong (2010): A dual Data Processing system using sensor Integrated RFID Middleware. Proceedings of the $2^{\text {nd }}$ International conference on Emerging Databases.

[3.] Noureddine Chikouche, Foudil Cherif \& Mohamed Bennohammed (2012): “An Authentication Protocol Based on combined RFID-Biometric system” In International journal of Advanced computer science and Application. Vol.3, No.4

[4.] Rashid Ahmed \& John N Avarissiotis (Retrieved 2015): Identification of Employees Using RFID in IE-NTUA

[5.] Steffan Elmstrom Holst Jensen \& Rune Hylsberg Jacobsen (Accessed 2015): Chapter 6: Integrating RFID with IP Host Identities” http://dx.doi.org/10.5772/53525 